Original Research Paper

\title{
Effects of Unilateral Cervical Vagotomy on Murine Dendritic Cells
}

\author{
${ }^{1}$ Daniel Sanzio Gimenes da Cruz, ${ }^{1}$ Ana Paula Nascimento de Lima, \\ ${ }^{2}$ Patrícia Benites Gonçalves da Silva, ${ }^{1}$ João Palermo-Neto and ${ }^{1}$ Cristina Massoco \\ ${ }^{I}$ Department of Pathology, School of Veterinary Medicine, University of Sao Paulo, Sao Paulo, Brazil \\ ${ }^{2}$ Department of Genetics and Evolutionary Biology, Institute of Biosciences, University of Sao Paulo, Sao Paulo, Brazil
}

\author{
Article history \\ Received: 14-03-2014 \\ Revised: 09-06-2015 \\ Accepted: 27-06-2015
}

Corresponding author:

Daniel Sanzio Gimenes da Cruz Department of Pathology, School of Veterinary Medicine, University of Sao Paulo, Sao Paulo, Brazil E-mail: dangimenes@usp.br

\begin{abstract}
The cholinergic anti-inflammatory pathway has been demonstrated to be an important mechanism that modulates several inflammatory diseases. This modulation occurs upon activation of the vagus nerve, which modulates splenic macrophages and lymphocytes through the predominant neurotransmitter, Acetylcholine (ACh). We hypothesized that this modulation could also take place in dendritic cells, as these cells represent a link between the innate and adaptive immune responses. Bone Marrow-Derived Dendritic Cells (BMDCs) were generated from mice subjected to a unilateral cervical vagotomy and the phenotypes and functions of the BMDCs were evaluated. No significant effects were found in BMDCs from vagotomized mice compared to cells from the control group. The specific immune response was evaluated with the Delayed-Type Hypersensitivity (DTH) skin test using Keyhole Limpet Hemocyanin (KLH) as an antigen and the peak of the response in the vagotomized animals was delayed compared to the control group. It is possible that the Dendritic Cells (DCs) can be modulated by the vagus nerve in other specific sites or under challenging conditions, but our work suggests that immune cells could be modulated by the components of another cholinergic anti-inflammatory pathway or by another neuro-immune pathway interaction.
\end{abstract}

Keywords: Dendritic Cells, Vagus Nerve, Cholinergic Anti-Inflammatory Pathway, Neuroimmunology

\section{Introduction}

The cholinergic anti-inflammatory pathway is an important neuro-immune communication mechanism in the maintenance of homeostasis. Specifically, the electrical or pharmacological stimulation of the vagus nerve modulates pro-inflammatory responses by binding its main neurotransmitter, Acetylcholine (ACh), into the nicotinic alpha-7 nicotinic receptors ( $\alpha 7 \mathrm{nAChR})$ present on immune cells (Pavlov and Tracey, 2005). Splenic macrophages are among these immune cells and are targets of the cholinergic antiinflammatory pathway in models of chronic intestinal disease (Ghia et al., 2006) and in endotoxemia, where the production and secretion of Tumor Necrosis Factor
(TNF) by these cells is inhibited (Borovikova et al., 2000). In lymphocytes, it has been shown that subdiaphragmatic vagotomy increases the production of pro-inflammatory cytokines and proliferation of these cells ex vivo (Karimi et al., 2010). Other immune cells such as Dendritic Cells (DCs) also possess cholinergic receptors (Kawashima et al., 2007), are involved in inflammatory processes and can activate other cells by making contact with receptors and secreting proinflammatory factors. Several in vitro studies have demonstrated the controversial effects of cholinergic agonists on these cells, sometimes showing antiinflammatory (Nouri-Shirazi and Guinet, 2003) or proinflammatory effects (Aicher et al., 2003; Gao et al., 2007; 2011). However, little is known about the 
effects of the vagus nerve on myeloid precursor cells or the subsequent phenotypic and functional outcomes of the Bone Marrow-Derived Dendritic Cells (BMDCs). In the present study, we aimed to determine the constitutive influence of the vagus nerve on the attributes of generated BMDCs using a partial vagotomy technique.

\section{Materials and Methods}

\section{Animals}

Eight to twelve week old female mice from the C57BL/6 and OT-II (animals with transgenic $\mathrm{T}$ cell receptors specific for ovalbumin-OVA) strains were used. The animals were housed in rooms with temperature $\left(22 \pm 2^{\circ} \mathrm{C}\right)$, humidity $(65-70 \%)$ and artificial light control (12 h light/12 h dark cycle, lights on at 7:00 am) in polypropylene cages with ad libitum access to Nuvilab ${ }^{\circledR}$ rodent chow (Nuvital, Colombo, PR) and filtered water. Sterile wood shavings were used as bedding for the animals. The mice were obtained from the Department of Pathology, School of Veterinary Medicine and Animal Science, University of Sao Paulo, where they were also maintained for all the experiments. The animals were used following the ethical standards and procedures related to use of laboratory animals from the FMVZ Ethics Committee-USP (2330/2011).

\section{Surgical Procedures}

The unilateral cervical vagotomy technique was based on and adapted from van Westerloo et al. (2005). Briefly, the animals were subjected to analgesia with a subcutaneous injection of $0.1 \mathrm{mg} \mathrm{kg}^{-1}$ of fentanyl and anesthetized intraperitoneally with ketamine and xylazine at doses of $0.05 \mathrm{mg} \mathrm{g}^{-1}$ and $0.02 \mathrm{mg} \mathrm{g}^{-1}$ body weight, respectively. A ventral cervical midline incision was made to expose the right vagal trunk, which was separated from the carotid artery and transected. The skin synthesis was performed with simple interrupted sutures using a 4-0 nylon thread. In the sham animals, the surgical intervention was similar to that used in the denervated group, but the nerve was not transected. Seven days after surgery, the animals were euthanized for the ex vivo experiments, or used in the in vivo experiment.

\section{Generation of Bone Marrow-Derived Dendritic Cells}

The method used to generate BMDCs was adapted from Lutz et al. (1999). Briefly, the bone marrow was taken from the femurs and tibiae of mice by washing the inside of the bones with RPMI-1640 medium (Gibco, Grand Island, NY) using sterile needles. The red blood cells were lysed with ACK lysis buffer $\left(\mathrm{NH}_{4} \mathrm{Cl} 0.15 \mathrm{M}\right.$, $\mathrm{KHCO}_{3} 10 \mathrm{mM}$, EDTA $\left.0.1 \mathrm{mM}\right)$. The cells were adjusted in a hemocytometer and maintained in dendritic cell culture medium (DC-RPMI), which consisted of RPMI-1640 medium supplemented with 10\% fetal bovine serum (FBS-LGC Biotechnology Cotia, SP), penicillin (100 $\left.\mathrm{U} \mathrm{mL}^{-1}\right)$ (Gibco, Grand Island, NY), streptomycin (100 $\left.\mathrm{mg} \mathrm{mL}^{-1}\right)$ (Gibco, Grand Island, NY), L-glutamine (Gibco, Grand Island, NY) (2 mM), 2mercaptoethanol (Gibco, Grand Island, NY) $(50 \mu \mathrm{M})$ and $3 \%$ supernatant of the NIH/3T3 fibroblast lineage containing the murine recombinant GM-CSF. The cells were seeded in a 24-well plate at a concentration of $7.5 \times 10^{5}$ cells/well and kept in a $5 \% \mathrm{CO}_{2}$ incubator at $37^{\circ} \mathrm{C}$. On the third day of culture, $1 \mathrm{~mL}$ of RPMI-DC was added to all wells. On the sixth day, half of the culture medium was removed from the wells, centrifuged, then the cells were resuspended in RPMI$\mathrm{DC}$ and returned to the plate. On the seventh day, to promote the maturation of the cells, $1 \mu \mathrm{g} \mathrm{mL}$ Lipopolysaccharide (LPS) was added during the last 24 $\mathrm{h}$ of culture.

\section{Assessment of Co-stimulatory Molecules by Flow Cytometry}

To measure the expression of co-stimulatory molecules, $1 \times 10^{6}$ BMDCs were incubated for $30 \mathrm{~min}$ at $4{ }^{\circ} \mathrm{C}$ in the dark with fluorescein isothiocyanate (FITC)-conjugated anti-CD11c (clone N418) (Biolegend), phycoerythrin (PE)-conjugated antiMHCII (clone AF6-120.1) (BD Biosciences), Allophycocyanin (APC)-conjugated anti-CD80 (clone 16-10A1) (BD Biosciences) and Peridinin Chlorophyll Protein (PerCP)-conjugated anti-CD86 (clone GL-1) (Biolegend). All antibodies were diluted 1:100 in PBS. The cells were washed once and the fluorescence was acquired using a FACSCalibur flow cytometer (Becton Dickinson).

\section{Endocytosis Assay by Flow Cytometry}

To assess the endocytic capacity of the BMDCs, $2 \times 10^{5}$ cells were incubated with the monoclonal antibody anti-CD11c (BioLegend, San Diego, CA) and killed Staphylococcus aureus were stained with Propidium Iodide (SAPI) for $1 \mathrm{~h}$ at $37^{\circ} \mathrm{C}$. The endocytosis was stopped by the addition of $3 \mathrm{mM}$ cold Ethylenediaminetetraacetic Acid (EDTA). The fluorescence was then acquired on a FACSCalibur flow cytometer (Becton Dickinson).

\section{In Vitro Stimulation of OVA-Specific T Cells}

After the generation of BMDCs, $1 \times 10^{6}$ cells $\mathrm{mL}^{-1}$ cells were incubated for $4 \mathrm{~h}$ with $1 \mathrm{mg} \mathrm{mL}^{-1}$ of grade 
II OVA (Sigma-Aldrich, St. Louis, $\mathrm{MO}$ ) in a $5 \% \mathrm{CO}_{2}$ incubator at $37^{\circ} \mathrm{C}$ for internalization of the protein. Then, $1 \mu \mathrm{g} \mathrm{mL}^{-1}$ of LPS was added for $24 \mathrm{~h}$ to induce maturation of the cells. Lymphocytes were collected and separated from the spleens of OT-II animals with Ficoll-Paque ${ }^{\mathrm{TM}}$ PLUS (GE Healthcare Life Sciences) according to the manufacturer's recommendations. The cells were then stained with Diacetate Succinimidyl Carboxyfluorescein Ester (CFSE) $(5 \mu \mathrm{g}$ $\mathrm{mL}^{-1}$ ) for $20 \mathrm{~min}$ at $37^{\circ} \mathrm{C}$. The cells were washed and plated at a concentration of $2 \times 10^{5}$ cells/well in coculture with the BMDCs at the following ratios of stained cells to BMDCs: 5:40,000; 25:8000; 50:4000; and 100:2000. The experiment was conducted in 96well $\mathrm{U}$ bottom plates, which were incubated in $5 \%$ $\mathrm{CO}_{2}$ at $37^{\circ} \mathrm{C}$ for 4 days. At the end of this period, the cells were collected and the fluorescence was acquired on a FACSCalibur flow cytometer (Becton and Dickinson). For the fluorescence data analysis, the percentage $(\%)$ of cell divisions was considered.

\section{Statistical Analysis}

All results were expressed as the means \pm SEM. The significant differences between groups were calculated using Student's $t$-test. The results were considered significant when $p<0.05$. The statistical analyses and the resulting graphs were generated using the Tree Star FlowJo software version 7.6.1 for Windows and GraphPad Prism version 6.0 for Windows.

\section{Results}

Right-sided Cervical Vagotomy does not Alter the Expression of Surface Molecules of BMDCs Neither Phagocytosis of SAPI

The expression of surface molecules such as MHCII and co-stimulatory molecules such as CD80 and CD86 are related to antigen presentation by DCs to naïve $\mathrm{T}$ cells. Therefore, we decided to investigate whether the right-sided cervical vagotomy (Vgx) was able to modify the expression of these molecules in BMDCs. Fig. 1a shows that the Vgx was not able to alter the expression of MHC-II and co-stimulatory molecules (CD80 and CD86) in the BMDCs treated with LPS in vagotomized animals relative to the sham controls. These results showed that the Vgx was not able to interfere with the generation (data not shown) and maturation of DCs. Still, it is important to consider that immature DCs have a greater endocytic capacity than mature cells (Banchereau et al., 2000). Therefore, we evaluated the ability of BMDCs derived from animals with $\mathrm{Vgx}$ to perform SAPI endocytosis. As shown in Fig. 1b, the fluorescent intensity of
$\mathrm{SAPI}^{+} \mathrm{DCs}$ in vagotomized animals did not differ from its sham counterparts. Because the results indicated that vagotomy did not have a significant effect on the function of immature BMDCs or their maturation, we decided to investigate whether the $\mathrm{Vgx}$ was able to alter the function of mature DCs.

Right-sided Cervical Vagotomy does not Alter the Ability of LPS-Stimulated DCs to Stimulate OVASpecific Proliferation of T Cells

One of the main characteristics of mature DCs is the stimulation of differentiation and proliferation of $\mathrm{T}$ cells. Thus, we investigated whether BMDCs of the animals receiving $\mathrm{Vgx}$ were able to stimulate a proliferative response in the OVA-specific T cells. To do this, the immature DCs were incubated with OVA, stimulated with LPS and co-cultured with T cells from OT-II mice. As shown in Fig. 2, the DCs were able to stimulate $\mathrm{T}$ cell proliferation in the 50 and 100 ratios, but no significant differences were found between the cells of the vagotomized animals compared to the sham group. These results showed that the vagotomy was not able to change either the function of BMDCs stimulated with LPS or their capacity to stimulate a T cell response.

The Right-Sided Cervical Vagotomy does not Alter the DTH Response but Interferes with the Kinetics of the Response

As one of our goals was to assess the antigen presentation of DCs in vagotomized mice, we used DTH as a model of antigen presentation in vivo because the DCs are important cells in the initiation of adaptive, antigen-specific cellular responses (Guinet et al., 2004). Fig. 3 shows that both group of animals presented paw edema starting at $24 \mathrm{~h}$ after the stimulus. Though the differences were not statistically significant, the profiles of the responses seemed to differ between the groups. Compared to the sham group, the Vgx group showed a smaller increase in swelling during the first $24 \mathrm{~h}$, which peaked at $48 \mathrm{~h}$ when the swelling of the sham animals was already decreasing. This result suggests that the kinetics of the inflammatory response were altered.

\section{Discussion}

The results obtained in our study demonstrated that the partial absence of the vagus nerve did not promote profound alterations in the phenotype or endocytosis of in vitro cultivated LPS-stimulated BMDCs. In contrast, there are some studies in the literature that 
points that nicotine, a cholinergic agonist, could promote diverse effects on BMDCs in vitro. One study has demonstrated that nicotine was able to elevate the expression of MHC-II, co-stimulatory molecules such as CD86 and CD40 and the adhesion molecules LFA-1 and CD54 in addition to reducing endocytosis (Aicher et al., 2003). Another study also reported a role for nicotine in stimulating DCs, which showed anti-tumor effects (Gao et al, 2007). Conversely, the opposite effects have been demonstrated (Guinet et al., 2004), with a reduction of the co-stimulatory markers and an increase of receptor-mediated endocytosis by nicotine-treated cells. It is possible that the stimulation of the $\alpha-7$ cholinergic receptors of the BMDCs could promote or inhibit DC maturation, but the reduction of cholinergic agonists used in our model does not necessarily lead to either effect.

Similarly to the results described above, we could not observe any alteration to the BMDC-induced $\mathrm{T}$ cell proliferation response. On the other hand, this has been observed by some other groups, who have reported either an increase (Aicher et al., 2003; Gao et al., 2007; 2011) or a decrease (Nouri-Shirazi and Guinet, 2003; Guinet et al., 2004) in lymphocyte proliferation induced by nicotine-stimulated DCs.
A
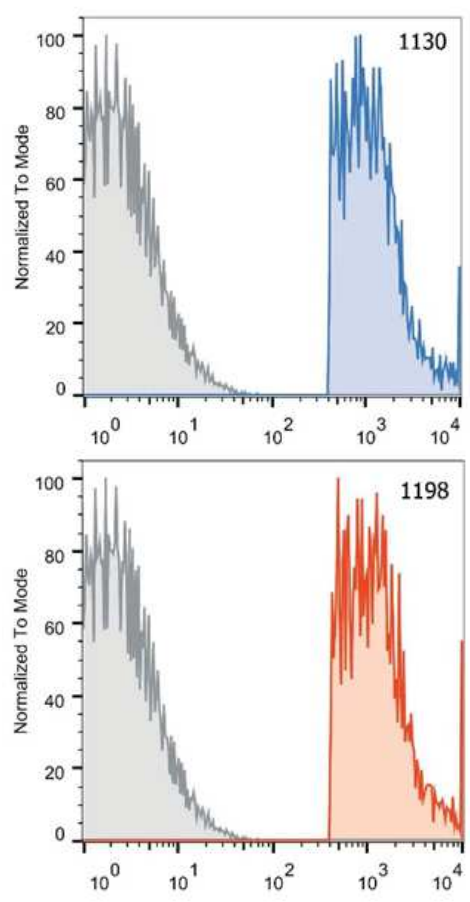

$\operatorname{CD} 86$
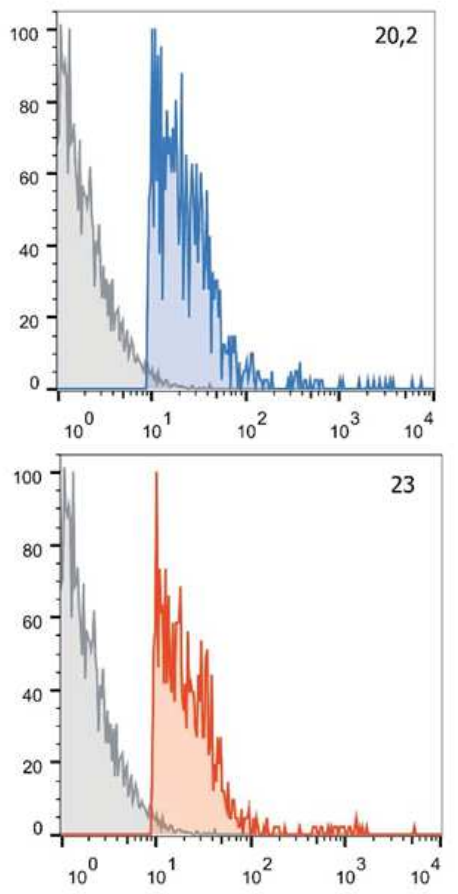

CD80

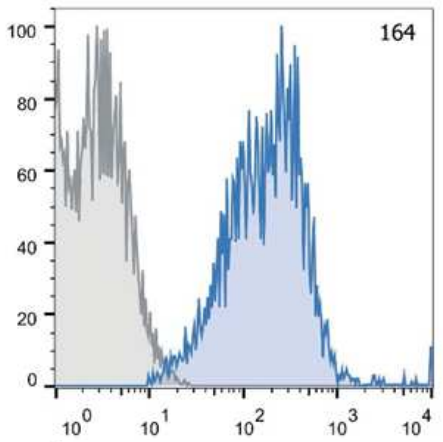

Sham

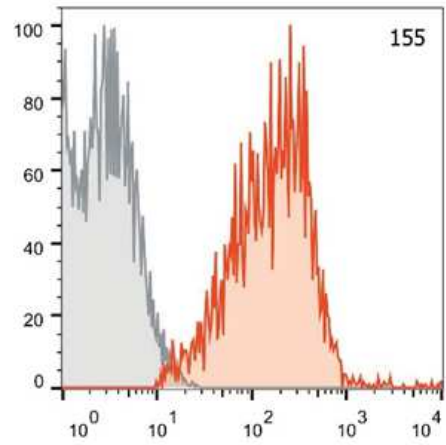

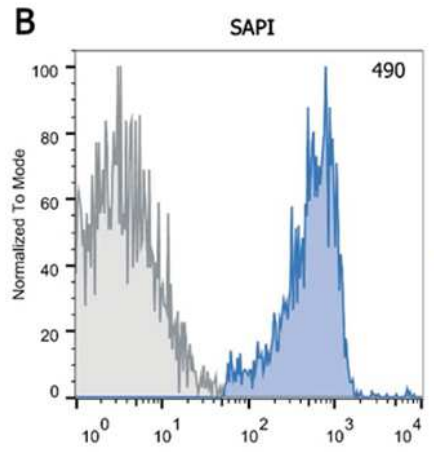
Sham
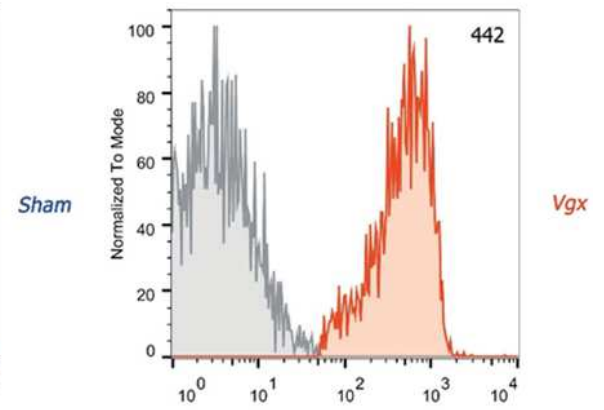

Fig. 1.(a) The Median Fluorescent Intensity (MFI) of MHC-II and the co-stimulatory molecules CD80 and CD86 and (b) the MFI of SAPI by BMDCs from mice subjected either to right-sided unilateral vagotomy (Vgx) or just exploratory surgery (sham). The light grey histogram represents an unstained control. The figures are representative of one of two independent experiments ( $\mathrm{n}=3$ animals/group/experiment) with similar results. The values are shown as the means \pm SEM of the samples ( $<<0.05$-Student's $t$ test) 


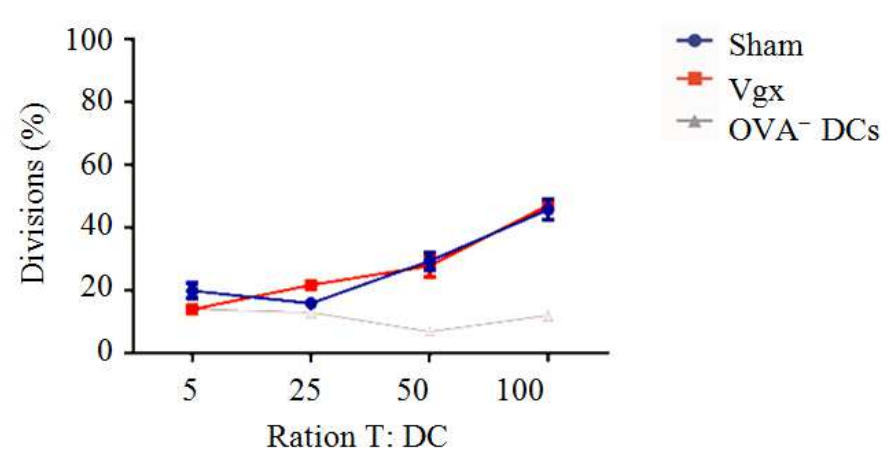

Fig. 2. Curve of the \% of divisions by OVA-specific T cells when co-cultured with BMDCs from mice subjected either to right-sided unilateral vagotomy (Vgx) or just exploratory surgery (sham). The figure represents one experiment ( $\mathrm{n}=10$ animals/group). The values are shown as the means \pm SEM of the replicates for each mouse $(\mathrm{p}<0.05$-Student's $t$ test)

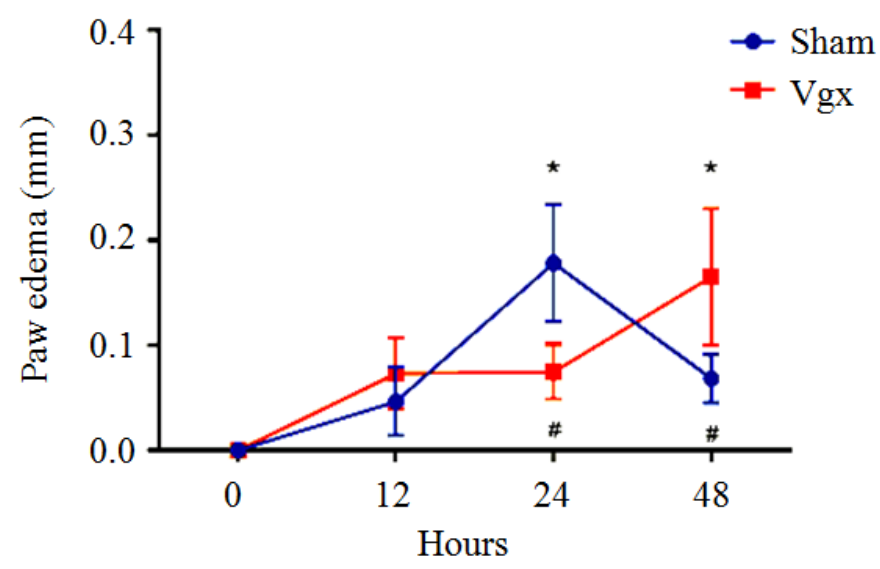

Fig. 3. A DTH curve depicting the magnitude of the swelling in the paws of mice subjected either to right-sided unilateral vagotomy (Vgx) or just exploratory surgery (sham) that were either immunized or challenged with KLH. The figure represents one of two independent experiments $(\mathrm{n}=3 \mathrm{animals} /$ group/experiment) with similar results. The values are shown as the means \pm SEM of the measurements. $\left({ }^{*}\right) \mathrm{p}<0.05 \mathrm{Vs}$ sham group, hour 0. (\#) $\mathrm{p}<0.05 \mathrm{Vs}$ Vgx group, hour 0 (Student's $t$-test)

The current literature documenting the effect of cholinergic agents on DCs as well as the relationship between these cholinergic agents, the vagus and DCs is controversial. Since the biological effects of nicotine or neurotransmitters is dependent on dose and exposure time, these controversial effects can be attributed to differences in experimental design, species, exposure time and particularly the levels of nicotine and/or neurotransmitters in contact with the DCs. This last point is especially true because DCs are finely controlled by nicotine and neurotransmitters in the local environment, as demonstrated in the work of $\mathrm{Hu}$ et al. (2012), in which different doses of nicotine followed by LPS provided different outcomes in DCs.

Most studies that have investigated the effects of cholinergic agents on DCs were performed with BMDCs treated with agonists in vitro. By contrast, our study was conducted with BMDCs that were influenced by the partial lack of innervation in vivo, making direct comparisons difficult per se. Taking the results of our in vitro studies together, we could speculate that the effects of the cholinergic pathway on DCs may occur on the tissues containing DCs and not on the precursors of these cells. In a study using a model of colitis, a subdiaphragmatic vagotomy was able to increase the expression of MHC-II and pro-inflammatory cytokines in $\mathrm{CD} 11 \mathrm{c}^{+}$cells in the spleen even though the vagotomy was not able to alter the expression of the co-stimulatory molecules CD80 and CD86 (Ji et al., 2014). This is a similar result to that obtained in the present study. Munyaka et al. (2014), using the same model of colitis, also showed an increase of pro-inflammatory cytokines produced by $\mathrm{T}$ cells co-cultured with spleen DCs from animals submitted to subdiaphragmatic vagotomy. In pathological situations, such as colitis, the vagus nerve may exert an anti-inflammatory tonic modulation on the dendritic cells of the spleen. However, it is possible that the effects of this tonic modulation are not strong enough 
to lead to significant changes in the BMDCs precursor cells, as the DCs generated from these cells showed similar phenotypes and functions to those from animals with intact vagus nerve.

In this study, we used the DTH test to further explore the possibility that the partial absence of the vagus nerve may interfere with the immunization process and the subsequent recall of this immunization. The DTH response is an inflammatory reaction in which a site challenged with an antigen that was used in a previous immunization is infiltrated by memory $\mathrm{T}$ lymphocytes and macrophages, which primarily mediate the inflammatory reaction (Szabo et al., 2003). In the present study, we used KLH with Freund's adjuvant as the antigen. Immunization is a fundamental process for the effective establishment of a DTH. In the immunization process, the antigenic agent is inoculated in the presence of an adjuvant that produces a local inflammatory response mediated by cells of the innate immune system. One of the populations of innate cells that are present at the site of this inflammation is the DCs (Banchereau et al., 2000). Once the DCs come into contact with the inoculated antigen, these cells migrate to the lymphoid organs and initiate antigen-specific adaptive cellular responses (Macia et al., 2006). As one of our goals was to evaluate the antigen presentation of DCs from vagotomized animals, it seemed relevant to use the DTH test as a model of antigen presentation in vivo. As a result, we expected that the vagotomized animals would have an exacerbated inflammatory response compared to the sham group, but this effect was not observed. Interestingly, the data tended to show that the peak response in the vagotomized animals was delayed and was possibly maintained for longer relative to the sham group, but these effects need further investigation using histological and cytokine studies because other immune cells may be involved.

In our study, the lack of observable effects in BMDCs cultivated from vagotomized mice and in our in vivo experiments could be perhaps explained by another mechanisms involved, such as the recovery time after surgery for animals that may be relevant to the cholinergic anti-inflammatory pathway. As demonstrated by Ghia et al. (2007), the effects of subdiaphragmatic vagotomy decrease over time and are eventually offset by other anti-inflammatory mechanisms, such as induction of Treg cells and the secretion of IL-10. Although our model of vagotomy was different, our experiments were performed a week after vagotomy, therefore, it is possible that these compensatory mechanisms were already activated in our mice. Moreover, as in unilateral cervical vagotomy, one of the cervical branches of the vagus nerve is preserved so it is not possible to rule out that this branch over time pass to exercise some of the functions of the branch transected and, therefore, the effects were not observable because the homeostasis was reestablished. We could hypothesize that only the acute activation of cholinergic receptors leads to more evident changes in DCs and that the tone of the vagal modulation on these cells causes only discrete biological effects, being shown only in the case of vagal activation front a signaling of the immune system.

Still, more recently was been demonstrated that instead of cholinergic, adrenergic agonists were able to increase endocytosis and IL-10 and reduce the production of IL-6, IL-12p70 and IL-23 in addition to polarize the $\mathrm{T}$ cell response for Tregs, suggesting that the effects on DCs are mediated by catecholamines and not by acetylcholine (Nijhuis et al., 2014). In fact, it is known that the vast majority of lymphoid organs, such as spleen and bone marrow, receive sympathetic innervations only and that the splenic nerve is an essential component of the cholinergic anti-inflammatory pathway. Martelli et al. (2014a) demonstrated that the splanchnic nerve and not the vagus, activate the splenic nerve producing anti-inflammatory responses. On another work, they hypothesized that communication between the vagus and the splenic nerve is not by nervous pathways, but by immune cells that are activated at sites of vagal innervation and migrate to the spleen where they release neurotransmitters that activate the sympathetic splenic fibers (Martelli et al., 2014b).

\section{Conclusion}

In summary, our results do not support the hypothesis of a constitutive modulation of the vagus nerve on the functional characteristics of BMDCs. However, we cannot dismiss that this modulation may occur at other sites, or in certain pathological situations. Moreover, studies that lead to a better understanding of the components involved in the cholinergic antiinflammatory pathway may provide clues concerning how this system can affect the behavior of DCs, either directly, or indirectly through other immune cells and factors secreted by those.

\section{Acknowledgment}

The authors would like to thank Dr. Alexandre Kanashiro for teaching the vagotomy procedures and Dr. Alexandre Basso for supplying the OT-II mice.

\section{Funding Information}

This study was supported by Fundação de Amparo à Pesquisa do Estado de São Paulo (FAPESP, Brazil), by thematic project $(09 / 51886-3)$ and study scholarship (2011/16261-2).

\section{Author Contributions}

Daniel Sanzio Gimenes da Cruz: Conducted all the surgical procedures and experiments, participated in the 
design of the study, statistical analysis, writing and the discussion of the manuscript.

Ana Paula Nascimento de Lima: Participated in the experiments, in the design of the study, writing and the discussion of the manuscript.

Patrícia Benites Gonçalves da Silva: Participated in the experiments, in the design of the study, writing and the discussion of the manuscript.

João Palermo-Neto: Participated in the design of the study, financial support and the discussion of the manuscript.

Cristina Massoco: Coordinator of the study, contributed to the design of the study, financial support, data analysis and the writing and discussion of the manuscript.

\section{Conflict of Interests}

The authors declare no conflict of interests.

\section{References}

Aicher, A., C. Heeschen, M. Mohaupt, J.P. Cooke and A.M. Zeiher et al., 2003. Nicotine strongly activates dendritic cell-mediated adaptive immunity: Potential role for progression of atherosclerotic lesions. Circulation, 107: 604-611.

DOI: 10.1161/01.CIR.0000047279.42427.6D

Banchereau, J., F. Briere, C. Caux, J. Davoust and S. Lebecque et al., 2000. Immunobiology of dendritic cells. Ann. Rev. Immunol., 18: 767-811.

DOI: 10.1146/annurev.immunol.18.1.767

Borovikova, L.V., S. Ivanova, M. Zhang, H. Yang and G.I. Botchkina et al., 2000. Vagus nerve stimulation attenuates the systemic inflammatory response to endotoxin. Nature, 405: 458-462.

DOI: $10.1038 / 35013070$

Gao, F.G., H.T. Li, Z.J. Li and J.R. Gu, 2011. Nicotine stimulated dendritic cells could achieve anti-tumor effects in mouse lung and liver cancer. J. Clin. Immunol., 31: 80-88. DOI: $10.1158 / 1078-0432 . C C R-07-0028$

Gao, F.G., F. Wan da and J.R. Gu, 2007. Ex vivo nicotine stimulation augments the efficacy of therapeutic bone marrow-derived dendritic cell vaccination. Clin. Cancer Res., 13: 3706-3712. DOI: 10.1158/1078-0432.CCR-07-0028

Ghia, J.E., P. Blennerhassett and S.M. Collins, 2007. Vagus nerve integrity and experimental colitis. Am. J. Physiol. Gastrointest. Liver Physiol., 293: G560567. DOI: 10.1152/ajpgi.00098.2007

Ghia, J.E., P. Blennerhassett, H. Kumar-Ondiveeran, E.F. Verdu and S.M. Collins, 2006. The vagus nerve: A tonic inhibitory influence associated with inflammatory bowel disease in a murine model. Gastroenterology, 131: 1122-1130. DOI: 10.1053/j.gastro.2006.08.016
Guinet, E., K. Yoshida and M. Nouri-Shirazi, 2004. Nicotinic environment affects the differentiation and functional maturation of monocytes derived dendritic cells (DCs). Immunol. Lett., 95: 45-55. DOI: 10.1016/j.imlet.2004.06.003

Hu, S.X., H.X. Sui, H.J. Jin, X.Y. Ni and X.X. Liu et al., 2012. Lipopolysaccharide and dose of nicotine determine the effects of nicotine on murine bone marrow-derived dendritic cells. Mol. Med. Rep., 5: 1005-1010. DOI: $10.3892 / \mathrm{mmr} .2012 .751$

Ji, H., M.F. Rabbi, B. Labis, V.A. Pavlov and K.J. Tracey et al., 2014. Central cholinergic activation of a vagus nerve-to-spleen circuit alleviates experimental colitis. Mucosal Immunol., 7: 335-347. DOI: $10.1038 / \mathrm{mi} .2013 .52$

Karimi, K., J. Bienenstock, L. Wang and P. Forsythe, 2010. The vagus nerve modulates CD4+ $\mathrm{T}$ cell activity. Brain Behav. Immun., 24: 316-323.

DOI: 10.1016/j.bbi.2009.10.016

Kawashima, K., K. Yoshikawa, Y.X. Fujii, Y. Moriwaki and H. Misawa, 2007. Expression and function of genes encoding cholinergic components in murine immune cells. Life Sci., 80: 2314-2319. DOI: $10.1016 /$ j.lfs.2007.02.036

Lutz, M.B., N. Kukutsch, A.L. Ogilvie, S. Rössner and F. Koch et al., 1999. An advanced culture method for generating large quantities of highly pure dendritic cells from mouse bone marrow. J. Immunol. Meth., 223: 77-92. DOI: 10.1016/S0022-1759(98)00204-X

Macia, L., M. Delacre, G. Abboud, T.S. Ouk and A. Delanoye et al., 2006. Impairment of dendritic cell functionality and steady-state number in obese mice. J. Immunol., 7: 5997-6006. DOI: $10.4049 /$ jimmunol.177.9.5997

Martelli, D., M.J. McKinley and R.M. McAllen, 2014a. The cholinergic anti-inflammatory pathway: A critical review. Auton. Neurosci., 182: 65-69. DOI: 10.1016/j.autneu.2013.12.007

Martelli, D., S.T. Yao, M.J. McKinley and R.M. McAllen, 2014b. Reflex control of inflammation by sympathetic nerves, not the vagus. J. Physiol., 592: 1677-1686. DOI: 10.1113/jphysiol.2013.268573

Munyaka, P., M.F. Rabbi, V.A. Pavlov, K.J. Tracey and E. Khafipour et al., 2014. Central muscarinic cholinergic activation alters interaction between splenic dendritic cell and CD4+CD25- T cells in experimental colitis. PLoS One, 10: e109272e109272. DOI: 10.1371/journal.pone.0109272

Nijhuis, L.E., B.J. Olivier, S. Dhawan, F.W. Hilbers and L. Boon et al., 2014. Adrenergic $\beta 2$ receptor activation stimulates anti-inflammatory properties of dendritic cells in vitro. PLoS One, 9: e85086e85086. DOI: 10.1371/journal.pone.0085086

Nouri-Shirazi, M. and E. Guinet, 2003. Evidence for the immunosuppressive role of nicotine on human dendritic cell functions. Immunology, 109: 365-373. DOI: $10.1046 / \mathrm{j} .1365-2567.2003 .01655 . \mathrm{x}$ 
Pavlov, V.A. and K.J. Tracey, 2005. The cholinergic anti-inflammatory pathway. Brain Behav. Immun., 19: 493-499. DOI: 10.1016/j.bbi.2005.03.015

Szabo, S.J., B.M. Sullivan, S.L. Peng and L.H. Glimcher, 2003. Molecular mechanisms regulating Th1 immune responses. Ann. Rev. Immunol., 21:713-758.

DOI: 10.1146/annurev.immunol.21.120601.140942 van Westerloo, D.J., I.A. Giebelen, S. Florquin, J. Daalhuisen and M.J. Bruno et al., 2005. The cholinergic anti-inflammatory pathway regulates the host response during septic peritonitis. J. Infect. Dis., 191: 2138-2148. DOI: 10.1086/430323 